\title{
Relative Nature of Thai Traditional Music through its Tuning System
}

\author{
Nattapol Wisuttipat \\ Bharat Vidyalaya School Srinakharinwirot University \\ email: nattapolwis@gmail.com
}

\begin{abstract}
Thai traditional music exhibits number of relative characteristics, though many of them are not expressed explicitly but only demonstrated orally. Its tuning system, despite having been put through many scientific studies for decades, cannot be given the absolute pitch frequencies. It is therefore possible that the tuning system does not have any absolute pitch frequencies but possesses relative nature. This papers aims to present the historic background, characteristics, past, current practices of Thai traditional music's tuning system and to put forward the theoretical idea of non-existence of absolute tuning frequency and that of relative nature of Thai traditional music's tuning system. The data was collected from previous researches on Thai traditional music's tuning system and from sample instruments. The latter was then compared to each other and analyzed with respect to the former. The preliminary results were that tuning system of Thai traditional music was in the form of seven-tone equal temperament without any sharps or flats. But practically, it was not exactly equal as musicians still prefer the traditionalbiased tunings which is believed to be more tuneful. Regarding the tuning practices, the tuning of Fine Arts Department are most dominating among several others. Even though, the tuning of Fine Arts Department measure from various sources are slightly different in terms of frequencies and intervals. It can be concluded that the tuning system of Thai traditional music does not rely on specific pitch frequency, but is relative to intervals and personal preferences and its absolute pitch frequency is yet to be established.
\end{abstract}

Keywords: Thai traditional music, tuning system, relative nature.

\section{INTRODUCTION}

The word Relative is defined in the Oxford Learner's Dictionary in the first entry as considered and judged by being compared with something else". The term is extensively used in various circumstances to suggest that something so described must be considered along with its surrounding contexts. Whether science's relative density, linguistics' relative pronouns or most of the terms associated with the adjective RELATIVE, it is an implication of reality that sometimes something can only be expressed when it is in connection with its ambience.

Musically, there are number of terms which are described using the word relative. Relative pitch, for an instance, is the ability of a person to identify or re-create a given musical note by comparing it to a reference note and identifying the interval between those two notes. A Relative minor key of Major C scale is another example. When considering the context under which the word relative is employed in various scopes, including music, we can transitively arrive at a preliminary conclusion that when something is designated with the word relative, it conveys the state of being connected to one other thing or more - of not being absolute. 
The traditional music of Thailand, as an embodiment of the country's musical culture, too exhibits its relative characteristics in several aspects.

Although Thai musicians do not expressively refer to these characteristics directly by the term itself, they are very well aware of its existence. This happens so because Thai traditional music is an oral tradition wherein the knowledge is being transmitted without much written evidence. The transmission could be in the form of either demonstration or oral explanation. As a result, Thai traditional musician perceive the existence of relative characteristics of Thai traditional music as an inexplicable, abstract concept whose comprehension can only be achieved by direct experience, i.e. playing Thai traditional music. Moreover, almost every writing dealing with Thai traditional music culture is in Thai and, more disappointingly, those written in other universal languages provide insufficient details. Combining these factors together, despite Thai traditional musician's fine perception of Thai traditional music's relative nature, the concept seems to be a very difficult concept to recognize and visualize even by native enthusiasts, let alone those foreign with Thailand's musical culture.

\section{MATERIALS AND METHODS}

One of unique musical aspects in Thai traditional music that hides the relative nature within is its tuning system. Tuning system of Thai traditional has developed its own system and process which have been being practiced since the ancient time to date. Basically, Thai musicians tune their instruments by listening and comparing the same note between reference instruments and the target instrument. There was no frequency measurement of the tuning system until it was calculated for the first time in England, 1885 by Ethnomusicologist pioneer Alexander J. Ellis and AlfredHipkinsduring an overseas exhibition of Thai musicians sent by King Rama V. Musically considering Thai traditional music as an exotic genre, since then, musical scholars have been very much interested in exploring more about Thai traditional music and were awakened to determining the exact calculation of its tuning system with several following sophisticated measuring experiments. Meanwhile in Thailand, though, musicians were too much occupied in performing aspects to give any attention to studying or calculating the tuning system. But there was PhraChenduriyang, a Thai band master who put forward the influential concept of tuning system of Thai traditional music in his somewhat brief writing that "As already stated, the Thai diatonic scale, is composed of seven full tones within its octave. These are evenly distributed in equidistant steps and there are no semitones between any of these full tone - steps." The statement was later proved to be inaccurate practically.

However, the urge to seriously study the tuning system of Thai traditional was realized only in 1967 after the initiative of His Majesty the King BhumibolAdulyadej triggered the first survey of pitch frequency of Thai traditional music followed by the second survey in 1994. The aim of both researches was to survey the pitch frequency of Thai traditional music and finalize a standard pitch frequency for Thai traditional music. The results obtained were very vast but they were inconclusive. There have also been plenty of similar researches that tried to find the right pitch frequency for Thai traditional music, only to add more tuning alternatives to already numerous 
existing tuning preferences without any sign of closing in on achieving standard pitch frequency. Today the effort of finding out the absolute value of pitch frequency, or even the issue of whether Thai traditional music have one, is still much debated.

Due to above circumstances, the writer is inspired by these obscurity shrouding Thai traditional music's tuning system that instead of establishing absolute pitch frequency for Thai traditional music, which, in high probability, could turn out to be futile, one should embrace the current phenomena of the pitch frequency diversity and support it by logically sound evidence. The writer also believes what is responsible for the diversity of tuning frequency and at the same time keeping them from deviating away from each other dramatically could be Thai traditional music's relative nature itself. This paper will, therefore, present the studied information about Thai traditional tuning system involving history, characteristics, past, current tuning practices in an analytical attempt to forward a theoretical idea of non-existence of absolute pitch frequency while validating the relative nature of Thai traditional music's tuning system in the process.

The objectives of the research

1. To present the historical background, characteristics, past and current practices of Thai traditional music tuning system.

2. To put forward a theoretical idea of non-existence of absolute tuning frequency and that of relative nature of Thai traditional music's tuning system.

Methods used.

This article follows the methodologies for quantitative research that proceeds with following steps

1. Establishing issue of study

2. Associated literature and related research reviews

3. Collecting relevant data

4. Analyzing obtained data with respect of available previous information.

5. Conclusions

\section{DISCUSSION}

Finding/Results

The research results, presented through descriptive analysis, will be discussed according to the following topics.

1. Tuning systems of Thai traditional music

1.1 Theoretical statement

1.2 Practical Situation

2. Past and Present Tuning Practice 
Results

1. Tuning systems of Thai traditional music

The tuning system of Thai traditional music is theoretically in the form of seventone equal temperament which incorporates only 7 tones in an octave. As a results, an octave of 1200 CENTS is evenly distributed in total 7 intervals instead of 12 and consequently altering the value of the twelve-tone equal temperament pitch intervals. Mathematically, each interval in seven-tone equal temperament is supposed to take the value of $1200 / 7=171.4$ CENTS. The comparison between Thai's seven-tone equal temperament and Western's twelve-tone equal temperament is shown below

Fig. 3 Comparison between the seven-tone equal temperament and the seven-tone equal temperament.

Twelve-tone equal temperament

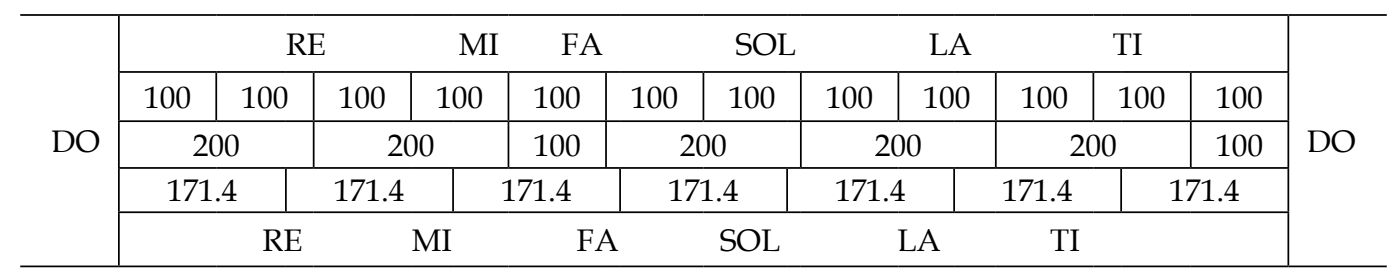

Seven-tone equal temperament

Although the intention is clear, in reality, the concept is notso perfectly implemented as it has been scientifically proven that the measured intervals of a functional eartuned Thai music instruments deviate more or less from the theorized value. However, the effect of the inaccuracy is not hugely felt as the principle of Thai traditional music relies on the pentatonic system, thus allowing more area of acceptability in tuning. As to the pitch frequency, it was found that the pitch frequency of Thai traditional music is not based on Western's musical system either and there has been no particular allusion of the pitch frequency of Thai traditional music.Nonetheless, some distant similarity between Thai traditional music and Western music in terms of pitch frequency can be observed. Oneresearch, under the initiatives of HM King Bhumibol, studied a pitch frequency of Thai traditional musical instruments which is nearest to the frequency of Western's C, i.e. 523.25 Hz.Sampling instruments from several famous musical ensembles are tested, giving the following results.

\begin{tabular}{|c|c|}
\hline ENSEMBLES & FREQUENCY (HZ) \\
\hline Fine Arts Department & 510.0 \\
\hline Royal Ensemble & 502.0 \\
\hline College of Dramatic Arts & 508.0 \\
\hline Pattyakosol & 500.0 \\
\hline Thong LorJu Wong & 509.53 \\
\hline Thammasat University & 516.84 \\
\hline Thai TV CH4 & 519.94 \\
\hline
\end{tabular}


It can be seen that none of the pitch frequencies of the above sampling instruments match that of the Western C. David Morton also mentioned about the pitch frequency of Thai traditional music that "there is no standard pitch level in Thailand".

\section{Past and Present Tuning Practice}

Before proceeding further, the writer feels the necessities to familiarize readers with the classifications of Thai melodic musical instruments as these classifications are going to be significantly related to the details of tuning practice later in this article. Firstly, Thai music instruments are classified, from the action by whichsound is produced, into 4 following types:

1. Plucked instruments, e.g. Chakay,

2. Bowed instruments, e.g. Saw U, Saw Duang and Saw Sam Sai

3. Struck instruments, e.g. RanadEk, RanadThum, Kong Wong Yai, Kong Wong Lek, RanadEk Leg and RanadThum Leg

4. Blown instruments, e.g. Pi, Klui

Second classification is based on difficulty of tuning. The Thai music instruments above can be reclassified into 3 types as follows

1. Permanently tuned Instruments, e.g. Klui, Pi, RanadEk Leg, RanadThum Leg

2. Fixed-tuning instruments, e.g. RanadEk, RanadThum, Kong Wong Yai and Kong Wong Lek, Chakhe

3. Free-tuning instruments, e.g. Saw Duang, Saw U, Saw Sam Sai. Chakhe In the second tuning difficulty-wise classification, the permanently tuned instruments are pre-tuned right from its makers and its pitch frequencies cannot be adjusted without the help of heavy machines. Therefore, the pitch frequencies of such instruments are less prone to fluctuation and hence preferred as reference instruments for tuning process. However, Pi and Khlui are not popularly used due to certain factors which can directly affect its pitch frequencies, e.g. the pressure of the air passing through the instruments or depth of the inserted mouthpiece. On the other hand, RanadEk Leg and RanadThum Leg are more preferable as they are almost independent of any internal and external factors. This means that these instruments produces the same pitch frequencies no matter how or whenever it is struck.

In the fixed tuning instruments, changing its pitch frequencies are relatively more convenient than the previous category. The pitch frequencies of these instruments can be altered by using a plasticine clay-like substance called "TA KUA". It is made of mixture of pure beeswax and lead powder. Having adhesive property, it is softened by heating before applying underneath the instruments. As it cools down, it hardens and sticks to the instruments. Its added weight will decrease the pitch of the applied instrument. For RanadEk and RanadThum, TA KUA is applied under each key and on both ends. For Kong Wong, TA KUA is pasted under each gong. The amount of TA KUA applied depends on the original weight of the instruments and the desired pitch, meaning that 2 identical pitches of 2 different instruments may not require the same amount of TA KUA applied. 
The last category, free tuning instruments, can be tuned and retuned right on the spot. The tuning of this kind of instruments is done using the mechanism within the instrument itself - turning knobs are an example - and without any external tools and help. Most of these instruments are stringed instruments. Thai musicians usually tune open strings of the instruments to the pitch of Klui of RanadEk. It is also to be noted that Chakhe, a Thai zither, seems to fall on both fixed-tuned and free-tuning instrument. Although its strings are tuned by merely adjusting its 3 protruding knobs, its frets, however, has to be pre-tuned to a desired temperament.

\section{Past Tuning Practice}

Thai musicians originally relied on their ears to adjust the pitches of an instrument to the desired frequencies. A tuning expert, usually a Thai music master or an experienced music maker, tuned every instrument in an ensemble to pitch frequencies that suit their ears the most. To do this, they must have reference instrument according to whose pitch frequencies other instruments are compared. The reference instruments are, therefore, those instruments that are part of the ensemble and whose pitch frequencies are durable and least susceptible to fluctuate. The reference instruments are either blown instruments such as Pi or Khlui, or metallic instruments such as Rand Leg, depending on types of ensemble played.

Back then Thai musicians did not pay much attention to the measurement precision of their tuning and also its definition. As a result, the pitch frequencies and intervals intervals of an instrument was never given a specific calculation. Later on, with advancement in theoretical and practical aspect of Thai music, the current Thai music tuning system is believed or understood to fall in a system that the modern scholars call it "seven equidistance" or "seven-tone equal temperament" or "seven-tone equal division of the octave".

There are 2 situations in which the reference pitch is determined. The first is when these instruments are being made, the maker tune them not by listening but merely using preset measurements of those instruments. These presets may be inherited from one's family or master. The second, with the help of heavy machines, is that a maker will fine-tune the finished permanently tuned instruments until its sound becomes most soothing to the maker's ears when the instruments are played.

Nonetheless, tuning preferences are different from one ensemble to another and even tuning preference of a single ensemble can differ from time to time, as they did not use any tools to record them. As a results, pitch frequencies of different ensembles of Thai music instruments can be slightly higher or lower than one another, which is, for Thai musicians, are acceptable as long as the whole ensemble is tuned uniformly. Some tuning preferences even go on to become an identity of an ensemble. Fine Arts Department, Royal Ensemble, College of Dramatic Arts, Pattyakosol, Thong LorJu Wong, Thammasat University, Thai TV CH4 are, as mentioned earlier, are examples of institutions whose ensemble is tuned with their own customization. 
Among these tuning instruments, it is the Fine Arts Department tuning that is dominating over other tunings through the process of cultural hegemony. Most of the active Thai musical ensembles and music instrument makers tend to adopt the Fine Arts Department tuning whilethe use of other tuning preferences nowadays is rather rare.

\section{Present tuning practice}

Today, how Thai instruments are tuned is quite varied in styles and methods. The most traditional methods of ear-tuning is still widely used today among Thai musicians and tuning experts. For KreuangSai ensemble, the instruments are usually tuned just before playing. By matching pitch of instruments to that of Khlui - the reference instruments - tuning the instruments in KhreungSai ensemble can be executed within a matter of minutes. The only exception is Chakhe whose bridges, as already mentioned, has to be set beforehand then only its pitch can be adjusted accordingly. Pipat Ensemble, however, demands more complicated endeavor. With reference instruments ready - in this case RanadLek - the fixed tuning instruments are tuned by removing/adding TA KUA. If a musician is not satisfied with the tuning of his instruments even after it has been tuned to the reference instruments, the musician will fine-tune the fixed tuning instruments to his/her satisfaction. This means that the RanadLek has to be retuned using heavy machine to match the musician tuning favors.

Even though the ear-tuning has been preceding and still active for several decades, this process is pretty much error prone - mostly from human, causing tuning inaccuracy. Keeping this drawback in mind, many Thai musicians and tuning experts now look for alternatives that offer more precise tuning measurement and at the same time reduce the risk of errors. So musicians and tuning experts alike endeavored to find a better alternatives than ear-tuning. Thai musician as well as tuning experts now turn to a more precise, reliable western chromatic electronic tuner with CENT/Hertz reader scale.

However, due to the fact the tuners and Thai music instruments follow different systems of temperament, these tuners cannot be used to tune to instruments directly, like using a meter scale to measure something in inches. Instead, these tuners are used to record the frequencies given out by Thai music instruments.

Although there are hundreds of different types of such electronic tuners available in market in terms of design, ergonomics, functionality, accuracy, but what matters most when it comes to tuning Thai music instruments is the least count of the tuner's reading scale since tuning system of Thai traditional music is interval-oriented. As tuning of Thai music instruments hardlyrefers to the absolute value on CENT scale, the tuner with smaller least count will be more favorable than that of larger least count. Sometimes a highly sophisticated electronic tuner that comes with many useful functions is neglected for tuning Thai music instruments than a relatively simple and cheaper electronic tuner, only because of least count difference. So it is the responsibility of a Thai musician or tuning experts to choose a suitable electronic tuner for one's tuning preference. 
The pitch frequencies of a finely ear-tuned fixed tuning instrument, most preferably RanatEk, are recorded using an electronic tuner. Its corresponding values are then noted down and will be applied to all other fixed tuning instruments. However, instead of registering entire pitch frequencies of an instrument, some prefer to record only one octave and use the obtained frequencies for subsequent tunings. This way the interval across an octave, if tuned correctly, is always maintained as 1200 CENTS. Again, some believe that by increasing or decreasing each pitch frequency slightly when moving up or down the octave, respectively, will make the instrument sound even more tuneful.

Since the Fine Arts Department tuning becomes the default tuning for Thai traditional music and the conventional method of tuning instruments by ears is being replaced by electronic tuner, the writer has collected 2 examples of Fine Arts Department tuning. The pitch are obtained froma finely-tuned instruments by recording it pitch by pitch using electronic tuner while keeping the reference pitch of the tuner at $A=440 \mathrm{~Hz}$.

Table 1.Table of Pitch Frequencies Courtesy of Mr. Un

$\mathrm{A}=440 \mathrm{~Hz}$

\begin{tabular}{|c|c|c|c|}
\hline Pitch & Intervals & Value (CENTS) & Intervals \\
\hline SOL & & $F-25$ & \multirow{2}{*}{155} \\
\hline LA & \multirow{2}{*}{170} & $\mathrm{~F} \#+30$ & \\
\hline $\mathrm{TI}$ & & G\# & \multirow{2}{*}{180} \\
\hline DO & \multirow{2}{*}{170} & A\# - 20 & \\
\hline RE & & $C-50$ & \multirow{2}{*}{180} \\
\hline MI & \multirow{2}{*}{160} & $C \#+30$ & \\
\hline FA & & D\# - 10 & 185 \\
\hline
\end{tabular}

As seen above, Table 1 shows the pitch frequency of an octave recorded from a finely tuned instruments. As mentioned earlier, there is only one note in an octave that falls on the absolute value of the tuner, i.e. G\#. There are variable pitch intervals in an octave ranging from 155 to 185. The SOL-FA intervals is the widest while the SOLLA intervals is the narrowest. There are reasons as to why the table starts with the note SOL and ends with the note FA. SOL is the starting of Ranad EK while FA is the last note of RanaadThum and Kong Wong. In this way a tuner tunes RanaadEk by looking down the table and tunes RanaadThum as well as Kong Wong by looking up the table. In this type of measurement, the pitch frequency is the same in every octave throughout an instrument. However, there is another type of tuning in which the pitch frequency is different across octaves. 
IJCAS: Vol. 2, Number 1 June 2015

Table 2:Table of Pitch Frequencies. Courtesy of Mr. SontornChangkleungkoon

\begin{tabular}{|c|c|c|c|c|c|c|c|c|}
\hline \multicolumn{3}{|c|}{ Ranat Thum } & \multicolumn{3}{|c|}{ RanatEk \& Kong Wong Yai } & \multicolumn{3}{|c|}{ Kong Wong Lek } \\
\hline \multirow{2}{*}{185} & $\begin{array}{c}1 \mathrm{C \#} \\
\text { (lowest) }\end{array}$ & Intervals & Intervals & - & intervals & Intervals & - & intervals \\
\hline & $2 \mathrm{D} \#-15$ & \multirow{2}{*}{180} & & - & & & - & \\
\hline \multirow{2}{*}{165} & $3 F-35$ & & \multirow{2}{*}{255} & $1 E-25$ & & & - & \\
\hline & $4 \mathrm{F \#}+30$ & \multirow{2}{*}{170} & & $2 \mathrm{~F} \#+30$ & \multirow{2}{*}{170} & & - & \\
\hline \multirow{2}{*}{170} & $5 \mathrm{G} \#$ & & \multirow{2}{*}{170} & $3 \mathrm{G} \#$ & & & - & \\
\hline & $6 \mathrm{A \# -30}$ & \multirow{2}{*}{170} & & $4 \mathrm{~A} \#-30$ & \multirow{2}{*}{170} & & - & \\
\hline \multirow{2}{*}{170} & $7 \mathrm{~B}+40$ & & \multirow{2}{*}{170} & $5 B+40$ & & & - & \\
\hline & $8 \mathrm{C \#}+10$ & \multirow{2}{*}{180} & & $\begin{array}{c}6 \mathrm{C} \#+ \\
10 \\
\end{array}$ & \multirow{2}{*}{180} & & - & \\
\hline \multirow{2}{*}{180} & $9 \mathrm{D \# -10}$ & & \multirow{2}{*}{180} & $\begin{array}{c}7 \mathrm{D} \#- \\
10 \\
\end{array}$ & & & - & \\
\hline & $10 F-30$ & \multirow{2}{*}{165} & & $8 F-30$ & \multirow{2}{*}{165} & & - & \\
\hline \multirow{2}{*}{170} & $11 \mathrm{~F} \#+35$ & & \multirow{2}{*}{170} & $9 \mathrm{~F} \#+35$ & & & - & \\
\hline & $12 \mathrm{G} \#+5$ & \multirow{2}{*}{170} & & $\begin{array}{c}10 \mathrm{G} \# \\
+5\end{array}$ & \multirow{2}{*}{170} & & - & \\
\hline \multirow{2}{*}{170} & 13 A\# - 25 & & \multirow{2}{*}{170} & $\begin{array}{c}11 \mathrm{A \#} \\
-25\end{array}$ & & & - & \\
\hline & $14 \mathrm{~B}+45$ & \multirow[b]{2}{*}{170} & & $\begin{array}{c}12 \mathrm{~B}+ \\
45 \\
\end{array}$ & & & $\begin{array}{c}1 \mathrm{~B}+ \\
45\end{array}$ & \\
\hline \multirow{2}{*}{180} & $\begin{array}{c}15 \mathrm{C} \#+ \\
15\end{array}$ & & 180 & $\begin{array}{c}13 \mathrm{CH}+ \\
15\end{array}$ & 170 & 170 & $\begin{array}{c}2 \mathrm{C} \#+ \\
15\end{array}$ & 180 \\
\hline & $16 \mathrm{D \# -5}$ & & 100 & $\begin{array}{c}14 \mathrm{D} \# \\
-5\end{array}$ & & & $\begin{array}{c}3 \mathrm{D} \# \\
-5\end{array}$ & 100 \\
\hline & $17 \mathrm{~F}-25$ & 180 & & $\begin{array}{c}15 \mathrm{~F}- \\
25 \\
\end{array}$ & 180 & 180 & $\begin{array}{c}4 \mathrm{~F}- \\
25 \\
\end{array}$ & \\
\hline & - & & 165 & $\begin{array}{c}16 \mathrm{~F} \#+ \\
40 \\
\end{array}$ & & & $\begin{array}{c}5 \mathrm{~F} \#+ \\
40 \\
\end{array}$ & 165 \\
\hline & - & & 170 & $\begin{array}{c}17 \mathrm{G \#}+ \\
10\end{array}$ & 170 & 170 & $\begin{array}{c}6 \mathrm{G} \#+ \\
10\end{array}$ & 170 \\
\hline & - & & $1 / 0$ & $\begin{array}{c}18 \mathrm{~A} \# \\
-20 \\
\end{array}$ & 170 & 170 & $\begin{array}{c}7 \mathrm{~A} \#- \\
20\end{array}$ & $1 / 0$ \\
\hline & - & & 170 & $\begin{array}{c}19 \mathrm{~B}+ \\
50\end{array}$ & $1 / 0$ & 170 & $\begin{array}{c}8 \mathrm{~B}+ \\
50\end{array}$ & 170 \\
\hline & - & & 170 & $\begin{array}{c}20 \mathrm{C \#}+ \\
20 \\
\end{array}$ & 180 & 180 & $\begin{array}{c}9 \mathrm{CH}+ \\
20 \\
\end{array}$ & 170 \\
\hline & - & & & $21 \mathrm{D \#}$ & & & $10 \mathrm{D} \#$ & \\
\hline & - & & 180 & $\begin{array}{c}22 \mathrm{~F}- \\
20\end{array}$ & & 165 & $\begin{array}{c}11 \mathrm{~F}- \\
20\end{array}$ & 180 \\
\hline & - & & & - & & & $\begin{array}{l}12 \mathrm{~F} \# \\
+45 \\
\end{array}$ & \\
\hline & - & & & - & & 170 & $\begin{array}{c}13 \mathrm{G \#} \\
+15 \\
\end{array}$ & 170 \\
\hline & - & & & - & & & $\begin{array}{c}14 \mathrm{A \#} \\
-15\end{array}$ & \\
\hline & - & & & - & & 175 & $\begin{array}{c}15 \mathrm{C}- \\
45\end{array}$ & 165 \\
\hline & - & & & - & & 173 & $\begin{array}{l}16 \mathrm{C \#} \\
+25 \\
\end{array}$ & 180 \\
\hline & & & & - & & 180 & $\begin{array}{l}\text { 17 D\# } \\
+5 \\
\end{array}$ & 180 \\
\hline & & & & - & & 180 & $\begin{array}{c}18 \mathrm{~F}- \\
15\end{array}$ & \\
\hline
\end{tabular}


Here, table 2 consists of 3 main columns of corresponding pitch frequencies and intervals for 4 different fixed tuning instruments, i.e. RanatThum, RanatEk, Khong Wong Yai and Kong Wong Lek. RanatEk and Kong Wong Yai share the same table, with Kong Wong Yai starting from the $6^{\text {th }}$ RanatEk bar. It can be observed that the frequencies of each note increase by 5 cents in every higher octave. However, the $1^{\text {st }}$ bar of RanatEk is tuned relatively lower than usual - E-25 instead of F-25. This enables RanatEk to combine intervals lower than the $8^{\text {th }}$ bar. It is also to be noted that although the there are changes in pitch frequency in different octaves, yet the pitch intervals of each octave remains constant. It is believed that by increasing each pitch frequency of a higher octave by 5 CENTS produces more soothing effect within an instrument. But, sometimes, when the same note of different distant octaves are simultaneously played, e.g. RanadEk's no. 5 and Kong Wong Lek no. 15, the dissonance becomes noticeable.

\section{CONCLUSION}

It is clear from the finding's numerical facts that the theory and practice of the tuning system of Thai traditional music is self-contradictory. The difference between the hypothetical and practical pitch interval is neither due to ignorance nor carelessness of Thai traditional musicians which would have resulted in irregularity of Thai traditional music, as suggested by some scholars. On the contrary, the phenomenon can be explained with historical and contextual evidence. Since Thai musicians originally tuned their instruments using their ears with the intention to divide the octave into 7 equal intervals, intervals of tuned instruments, as inspected by many scholars, appeared to be slightly deviated from the ideal value. This case was understandable, considering inconsistency of human ears.

Later on, technological advancements finally have allowed us to correctly tune Thai musical instruments according to the 7 tone equal temperament, which is 171.4 cents apart. Nevertheless, Thai musicians are already so accustomed to listening to the conventional biased tuning that such tuning is thought to be the most pleasant and that tunings other than that is regarded as non-melodious. An attempt to convince Thai musicians to use the real 7 tone equal temperament may pave the way to more consolidated version Thai music. But, on the other hand, it may prove to be a difficult and time-consuming task.

Regarding the pitch frequencies of Thai traditional music instruments, from the very first analysis of the instruments which have been sent for an exhibition in Europe to the modern extensive examination of Thai traditional music instruments across the country, whether or not does the absolute pitch frequency of Thai traditional music exist is still a controversy. The more instruments surveyed, the more tuning preferences added to the list. Today, it is agreed upon by every Thai musician that the pitch frequencies of the Fine Arts Department is the most reliable one. However, the 2 different tables of pitch frequencies in the previous section instruments show that almost every corresponding pitch frequencies are more or less different from one another despite the claim that all follow the Fine Arts Department tuning. The use of electronic tuners has let Thai musicians as well as music makers come up with their own Fine Arts Department tunings. 
Apparently, there is no officially-determined standard pitch frequency. So, regardless of tuning methods, a music ensemble, group or school tune their instruments using their own pitch frequencies. As a result, it is possible that the pitch frequencies of one ensemble, groups, and schools can be notably higher or lower than the others. However, Thai musicians seem to neglect these facts and have no difficulty playing in musical ensembles with different tuning finish, as long as the tuning of each and every instruments of the whole ensemble is maintained uniformly. No matter how the tuning perspectives are viewed, the establishment of an absolute pitch frequency of Thai traditional music is far from conclusion.

On the other hand, if we look back at the purpose or the intention of tuning Thai musical instruments, it has been always to divide the octave into 7 equal intervals then and now, whatever the preferences may be, regardless of how it may be done. With that said, it is therefore reasonable enough to assume that it is the intervals that play more significant part than the frequencies when it comes to tuning Thai traditional musical instruments.And that makes the tuning system of Thai traditional music relative or it is interval-oriented. As the reference frequencies are subjective from one's preference to another, but the intervals are always supposed to be constant. In practice, however, the intervals, and frequencies, are not so exactly identical throughout an octave and across ensembles due tofew reasons. First is the limit of electronic tuners as Thai traditional music tuning does not only refer to the absolute value on the reading alone and electronic tuners nowadays come with various least count values. Second is the familiarity of the conventional biased tunings. On the other hand, it is the conventional tuning that maintains the close range of pitch frequency of a single note and also prevents it from further expansion.

The relative nature of Thai traditional music can also explain the fact of the tuning diversities of Thai traditional music from past to present and can also account for the dissimilarities of the tunings of different ensembles. In spite of that, Thai musicians do not mind playing instruments whose tuning they are not accustomed of.

It is also noteworthy that relative nature of Thai traditional music is also surprisingly parallel to that of Thai language. Being tonal in nature, Thai language is communicated through precise pronunciation of relative intonations. The same word, if pronounced with different intonation, would have different meaning and a mistake in intonating words would change their meaning or could indicate the native habitat of a speaker. Also, a word of single intonation might have different meaning if it is heard along with different surrounding words.

\section{Suggestions}

With today technology advancing by leaps and bounds, there is no denying that smartphones is becoming indispensable to human life. But these gadgets too have certain positive impacts on the musical domain. Various mobile tuning applications available across different operating platforms through respective online App Store, both for free and paid, has made tuning even more so affordable and accessible than ever. Moreover, some of these useful applications offer an incredible precision with 
very small least count of 1 CENT or even in decimals. In the future, these technological advancement is going to contribute to making Thai music instruments tuning so accurate and easily feasible that everybody can effectively execute just by launching an application within couple taps. The day might not be so far away that a standardized pitch frequencies of Thai music instruments with ideal interval of 171.4 CENT can be achieved realisticallyor perhaps there could be a tuner particularly made for Thai instruments tuning in the near future.

Although there was not much sound evidence regarding the historic background of Thai traditional music tuning system, there is no denying that the development of tuning system has seen steady developing, both by the Thai musicians and by external assistance. This may be a tiny progress within a large system, but it proves that Thai music society is not a stagnant culture waiting to crumble on itself. On the contrary, it is rather a dynamic phenomenon that has been well taken care of by its people to ensure that it thrives with the ever changing world without losing its identity and at the same time open to the interested people from any cultures to learn and share.

\section{REFERENCES}

Chulalongkorn University. (1999). The Study of Pitch Frequency of Thai Traditional Music - An HM the King Bhumibol's Initiative. Bangkok. RueanKaew Press.

Morton, David. (1976). The Traditional Music of Thailand. University of California Press, Berkeley and Los Angeles.

Wisuttipat, Manop. (2015). “Theory and Practice on Thai Music Tuning System”. DontriPriprem, vol. 5. Bangkok. Santisiri Press.

\section{ONLINE RESOURCES}

British Council. "Relative Pronouns". English Grammar. July 1, 2015. https:// learnenglish.britishcouncil.org/en/english-grammar

Nave, Rod. "Relative Humidity". HyperPhysics Concepts. July 1, 2015. http:// hyperphysics.phy-astr.gsu.edu/hbase/kinetic/relhum.html

Oxford University. "Relative Adjective - Definition, Pictures, Pronunciation and Usage Notes | Oxford Advanced Learner's Dictionary at OxfordLearnersDictionaries. com". Oxford Learner's Dictionaries. July 1, 2015. http://www. oxfordlearnersdictionaries.com/definition/english/relative_1?q=relative

Thassuya. "Pitch Frequencies of 7 equal temperament in Thai Classical Music". Thai Classical Experimental Music. March 12, 2013. June 28, 2015. http://thassuya. blogspot.com/2013/03/7.html

\section{OTHERS}

Changkleungkul, Soontorn. (2011). Interview by ManopWisuttipat.. 